\title{
Effects of Tension and Edge Deflection upon the Surface Accuracy of a Rectangular Membrane of a Space Reflector
}

\author{
By Sumio Kato \\ Aerospace Company, Kawasaki Heavy Industries, Ltd., Kakamigahara, Japan
}

(Received March 29th, 2006)

\begin{abstract}
The surface accuracy of a rectangular membrane subjected to anisotropic tension in a reflector surface is investigated. The rectangular membrane is assumed to be located at an arbitrary point on the reflector surface with an arbitrary rotation angle $\alpha$ between the side of the membrane and the direction of the principal curvature of the reflector surface. The deflection and surface error of the membrane are calculated based on an analytical solution of the linear membrane equation. Three kinds of rectangular optimum membranes, whose surface errors are minimized with respect to parameters that specify the edge deflections, are considered and evaluated. These surface errors, $\delta_{1}$ (equal-curvature-edge-optimum membrane), $\delta_{2 \text { opt }}$ (optimum-2 membrane), $\delta_{3}$ (optimum-3 membrane), are also compared with $\delta_{\text {copt }}$ (surface error of a coincident-optimum-edge membrane) that is studied in other literature. In a certain range of parameters, it is found that the appropriate tension ratio reduces the surface error of each optimized membrane as compared to isotropic tension. The more slender the membrane is and the higher the ratio of tension (ratio of higher tension on the longer side to lower tension on the shorter side) is, the smaller the surface error becomes. The minimum surface error is obtained when the longer side is placed parallel to the direction of the larger principal curvature at the location and the longer side is loaded with higher tension. At $\alpha=0, \delta_{2 \text { opt }}$ is equal to $\delta_{3}$. As for a slender membrane whose longer side is loaded with higher tension, $\delta_{2 \text { opt }}\left(=\delta_{3}\right)$ is approximately equal to $\delta_{1}$ and $\delta_{\text {copt }}$ at $\alpha=0$, and $\delta_{2 \text { opt }}$ $\left(\cong \delta_{\text {copt }}\right)$ is smaller than $\delta_{1}\left(\cong \delta_{3}\right)$ at $\alpha \neq 0$. It is found that $\delta_{2 \text { opt }}$ is the smallest among other membranes, and the surface error is about 0 $-15 \%$ less than $\delta_{\text {copt }}$. Each surface error decreases as the distance from the vertex increases.
\end{abstract}

Key Words: Surface Error, Rms Error, Mesh-Type Reflector, Tension Ratio, Rectangular Membrane, Space Reflector

\section{Nomenclature}

$2 a, 2 b$ : length and width of a rectangular membrane (see Fig. 2(b))

APS: approximate parabolic surface

$F$ : focal length of an ideal parabolic surface

g: $1+k^{2}\left(X_{0}^{2}+Y_{0}^{2}\right)$

$h$ : amount of translation in the $\zeta$ axis direction

$H: F h /(4 a b)$

$k: 1 / R$

$k_{1}, k_{2}$ : principal curvatures at $P_{0}$

$K_{1}, K_{2}: F k_{1} /(4 \lambda), F k_{2} /(4 \lambda)$

$l_{1}, l_{2}$ : principal curvatures of a parabolic surface for the expression of $w_{\mathrm{B}}$ (Eq. (5b))

$L_{1}, \quad L_{2}: \quad \mathrm{Fl}_{1} /(4 \lambda), \mathrm{Fl}_{2} /(4 \lambda)$

$O-X, Y, Z: \quad$ orthogonal cartesian coordinate system (see Fig. 2(a))

$P_{0}$ : arbitrary point on an ideal parabolic surface (see Fig. 2(a))

$P_{0}-\xi, \eta, \zeta:$ local coordinate system at $P_{0}$ on the ideal parabolic surface (see Fig. 2(a))

$P_{0}-x, y, \zeta:$ local coordinate system at $P_{0}$ obtained by rotating the $\xi$ and $\eta$ axes around the $\zeta$ axis with an angle of $\alpha$ (see Fig. 2(b))

$P_{0}-x^{*}, y^{*}, \zeta:$ local coordinate system at $P_{0}$ obtained by rotating the $x$ and $y$ axes around the $\zeta$ axis with an angle of $\beta$

(C) 2006 The Japan Society for Aeronautical and Space Sciences (see Fig. 2(b))

rms: root-mean-square

$r_{1}, r_{2}$ : principal radii of curvature at $P_{0}$

$R$ : radius of curvature at the apex of an ideal parabolic surface

$T_{x} T_{y}$ : constant tensile forces per unit length loaded on the sides of a rectangular membrane (see Fig. 2(b))

$w$ : deflection of a membrane

$w_{\mathrm{B}}$ : deflection of the edges of a membrane

$(x, y, \zeta)$ : coordinates in the $P_{0}-x, y, \zeta$ system

$\left(X_{0}, Y_{0}, Z_{0}\right):$ coordinates of $P_{0}$ in the $O-X, Y, Z$ system

$y^{\prime}: y / \kappa$

$\alpha$ : rotation angle of the $x$ axis from the $\xi$ axis (see Fig. 2(b))

$\beta$ : rotation angle of the $x^{*}$ axis from the $x$ axis (see Fig. 2(b))

$\delta_{\text {rms }}: \quad$ surface error (Eq. (7))

$\delta_{1}: F \delta_{\text {rms }} /(4 a b)$ of an equal-curvature-edge-optimum membrane

$\delta_{2}: F \delta_{\text {rms }} /(4 a b)$ of a two-curvature membrane

$\delta_{2 \text { opt }}: \quad F_{\delta_{\text {rms }}} /(4 a b)$ of an optimum-2 membrane

$\delta_{3}: F \delta_{\text {rms }} /(4 a b)$ of an optimum-3 membrane

$\delta_{\mathrm{c}}: F_{\delta_{\text {rms }}} /(4 a b)$ of a coincident-edge membrane of Ref. 19)

$\delta_{\text {copt }}: F \delta_{\text {rms }} /(4 a b)$ of a coincident-optimum-edge membrane of Ref. 19)

$\kappa:$ tension ratio parameter, $\left(T_{y} / T_{x}\right)^{1 / 2}$

$\lambda: b / a$ 
Trans. JSASS Space Tech. Japan Vol. 4 (2006)

\author{
$\lambda^{\prime}: \lambda / \kappa$ \\ $\zeta_{\mathrm{APS}}:$ APS at point $P_{o}$ \\ Subscripts \\ max: maximum with respect to $\beta$ \\ min: minimum with respect to $\beta$
}

\section{Introduction}

Parabolic surface structures are fundamental for space reflector technology such as solar reflectors and space antennas. As a surface structure for large space reflectors, three types of structures are proposed: solid ${ }^{1,2)}$, mesh $^{2-7)}$ and inflatable types. ${ }^{8-13)}$ In the case the reflector structure is used for antenna applications, it is very important to obtain a highly accurate reflection surface. In the case of a mesh-type reflector, one of the typical surface structures is a membrane element (mesh element) with the edges (the boundaries) supported by a rigid structure. The surface error of the membrane depends on parameters such as the size, shape (square, rectangle, equilateral triangle, etc.), edge deflection, location in the reflector surface, tension ratio and so on. ${ }^{14-19)}$

An example of a rectangular membrane that constitutes the reflector surface is shown in Fig. 1. For such a reflector, it is apparent that the rotation angle $\alpha$ between the side and the direction of the principal curvature of each membrane is different. Usually membranes are treated subjected to isotropic tension; however, in a certain range of parameters, the appropriate tension ratio may result in smaller surface error as compared to isotropic tension. ${ }^{17-19)}$ For the design of a reflector structure, as shown in Fig. 1 , with high surface accuracy, it is very important to understand the relation between the surface accuracy of the membrane and the parameters, including tension ratio parameter, edge deflection, rotation angle, aspect ratio, and location in the reflector surface.

Fitcher ${ }^{14)}$ investigated the effects of curved edges with parabolic deflections upon the surface error of equilateral triangular and rectangular membranes subjected to isotropic tension placed at the vertex of a reflector surface.

Kato and Natori ${ }^{15)}$ studied the effects of curved boundaries, the shape, size and position in a reflector surface, rotation angle about the normal axis, etc. upon the surface error of a circular membrane element and an equilateral triangular membrane element subjected to isotropic tension. In a similar way, Kato and Matsuzaki ${ }^{16}$ studied the effects of these parameters upon the surface error of a rectangular menbrane element subjected to isotropic tension located at an arbitrary point on a reflector surface.

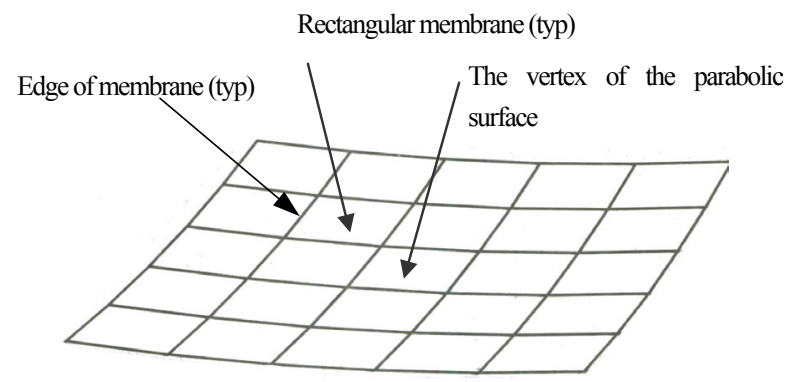

Fig. 1. Rectangular membrane that constitutes the reflector surface (example).
Furuya and Miura ${ }^{17,18)}$ investigated the effects of the tension ratio parameter and aspect ratio upon the surface error of a rectangular membrane located at an arbitrary point on a reflector surface. The edges of the rectangular membrane are assumed to coincide with the best-fit parabolic surface. The membrane element is placed so that the sides are parallel to the directions of the principal curvatures of the parabolic surface. The basic effectiveness of anisotropic tension upon the surface accuracy of a rectangular membrane is demonstrated. However, the investigation is not sufficient for the design of such a reflector in that $\alpha$ is limited to a value of zero, and the edge is fixed on the best-fit parabolic surface.

Kato ${ }^{19)}$ investigated the effects of tension ratio and aspect ratio upon the surface accuracy of a rectangular membrane with two kinds of edge deflections located at an arbitrary point on a reflector surface with an arbitrary angle $\alpha$ : a coincident-edge membrane and a coincident-optimum-edge membrane. A coincident-edge membrane is defined such that the edges coincide with the approximate parabolic surface (APS) of the local point where the membrane is placed. A coincident-optimum-edge membrane is obtained by minimizing the rms error using the normal translation of a coincident-edge membrane. The study showed that the surface error of a coincident-optimum-edge membrane is about $0.4-0.5$ of a coincident-edge membrane and the effectiveness of the anisotropic tension with edge deflection in the normal direction is demonstrated. However, the edge deflection is limited in that the edges are on the APS or translated in the normal direction. Different edge deflections may improve the surface accuracy of the membrane.

In this paper, the surface accuracy of a rectangular membrane, which is subjected to anisotropic tension and located at an arbitrary point on a reflector surface with an arbitrary rotation angle $\alpha$ is investigated. Rectangular membranes with three kinds of optimized edge deflections are considered: equal-curvature-edge-optimum, optimum-2 and optimum-3 membranes. The equal-curvature-edge optimum membrane is given by specifying equal curvatures on all edges, and minimizing the surface error with respect to the curvature and normal translation. The optimum- 2 membrane is obtained by minimizing the surface error with respect to $L_{1}, L_{2}, H$ and $\beta$ (parameters that effect the edge deflection). The optimum-3 membrane is given by specifying $\beta=0$ and minimizing the surface error with respect to $L_{1}, L_{2}$ and $H$. By introducing a new coordinate parameter, the linear differential equation of membrane deflection subjected to anisotropic tension becomes the equation of membrane deflection subjected to isotropic tension, Laplace's equation, defined in a transformed region. The deflection of the membrane subjected to isotropic tension is solved analytically, and is used to obtain the membrane deflection and surface error subjected to anisotropic tension. The formulation includes the parameters of $L_{1}, L_{2}$ and $\beta$, which are not treated in Ref. 19). Surface error minimization with respect to parameters that effect the edge deflection is carried out. The effects of various parameters such as tension ratio parameter $\kappa$, aspect ratio of the rectangular membrane $\lambda$, rotation angle $\alpha$ and location $g$ upon each surface error of three kinds of optimized rectangular membranes are analyzed. Each surface error of these optimum membranes is compared with each other, and is also compared with that of the coincident-optimum-edge membrane treated in Ref. 19). Then the effectiveness of the method to reduce the surface error of a rectangular membrane using anisotropic tension with optimized edge deflections different from those treated in Ref. 19) is evaluated. 
S. KATO: Effects of Tension and Edge Deflection upon the Surface Accuracy of a Rectangular Membrane of a Space Reflector

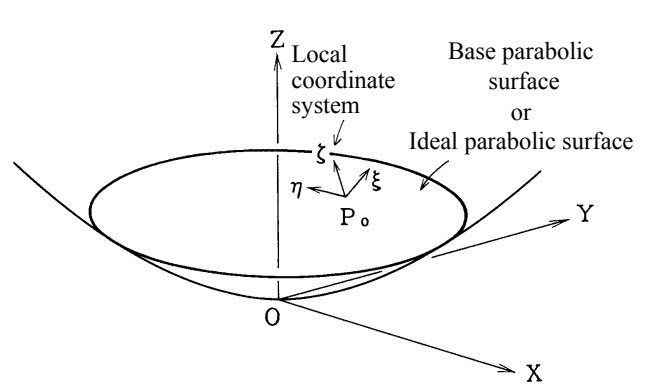

Fig. 2(a). Base parabolic surface and local coordinate system.

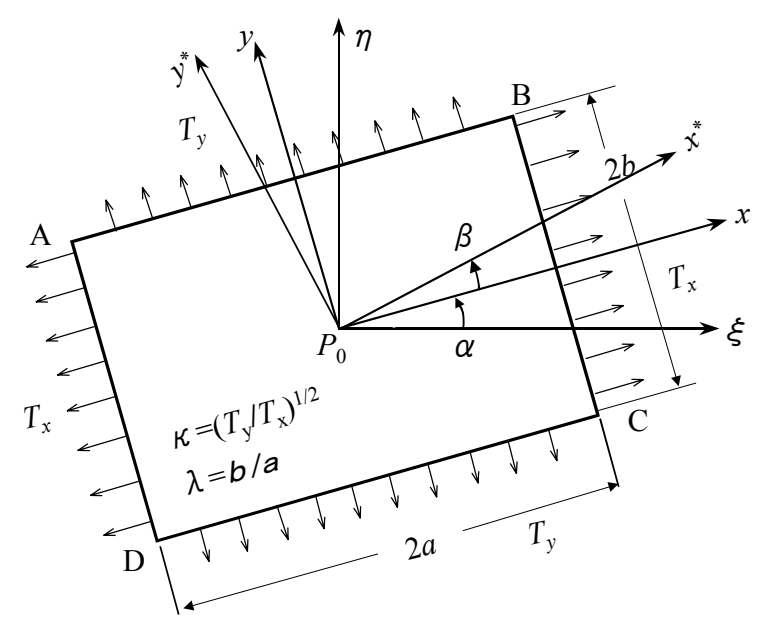

Fig. 2(b). Rectangular membrane.

In the present study, the surface accuracy of a membrane with respect to various parameters is brought into focus. In the design of a reflector that consists of numerous membranes, the surface error of the entire reflector is important. Among parameters that influence the surface accuracy of a membrane, parameters such as $\alpha, \lambda$ and $g$ are constrained by those of neighboring membranes when membranes are arranged in the reflector. This means that the surface error minimization of each membrane with respect to these parameters being independent of other membranes is impossible. On the other hand, $\kappa$ and parameters that effect the edge deflections, which also influence the surface accuracy of a membrane, are not constrained by the parameters of neighboring membranes, thereby allowing the surface error minimization of each membrane to be carried out independently of the neighboring membranes. The constraints with respect to the parameters mentioned above affect the surface error minimization of the entire reflector.

The analytical formulation and results obtained in this paper will be useful for designing rectangular membranes subjected to the anisotropic tension of large space reflectors.

\section{Ideal and Approximate Parabolic Surfaces}

A parabolic surface with the focal length of $F$ is considered, as is shown in Fig. 2(a). The point $O$ is the vertex of the surface, and the $Z$ axis is the center axis of the surface. In this paper, this parabolic surface is called the "base parabolic surface" or the "ideal parabolic surface", which is expressed by the following equations:

$$
\begin{aligned}
Z= & \left(X^{2}+Y^{2}\right) /(4 F)=\left(X^{2}+Y^{2}\right) /(2 R) \\
& =(k / 2)\left(X^{2}+Y^{2}\right)
\end{aligned}
$$

Consider a local coordinate system at a point $P_{0}$ on the ideal parabolic surface, $P_{0}-\xi, \eta, \zeta$, where $P_{0}$ is the origin. The axes $\xi$ and $\eta$ are tangent to the lines of principal curvature with a smaller value and a larger value, respectively, and the axis $\zeta$ is normal to the surface (see Figs. 2(a) and 2(b)). Using the local coordinate system, the ideal parabolic surface in the neighborhood of $P_{0}$ is expressed by the following equation:

$$
\begin{aligned}
\zeta(\xi, \eta)= & a_{00}+a_{10} \xi+a_{01} \eta+a_{11} \xi \eta \\
& +a_{20} \xi^{2}+a_{02} \eta^{2}+a_{30} \xi^{3}+a_{21} \xi^{2} \eta+\cdots
\end{aligned}
$$

where

$$
\begin{aligned}
& a_{00}=\zeta(0,0), a_{10}=\partial \zeta(0,0) / \partial \xi, a_{01}=\partial \zeta(0,0) / \partial \eta \\
& a_{11}=\partial^{2} \zeta(0,0) / \partial \xi \partial \eta, a_{20}=(1 / 2) \partial^{2} \zeta(0,0) / \partial \xi^{2}, \\
& a_{02}=(1 / 2) \partial^{2} \zeta(0,0) / \partial \eta^{2}, \cdots
\end{aligned}
$$

By leaving terms of 0 to 2 nd order with respect to $\xi$ and $\eta$, and considering the coefficients excepting $a_{20}$ and $a_{02}$ are zero, we can approximate the ideal parabolic surface by $\zeta_{\mathrm{APS}}$, which is expressed by the following equation:

$$
\zeta_{\mathrm{APS}}=(1 / 2)\left(k_{1} \xi^{2}+k_{2} \eta^{2}\right)
$$

where

$$
\begin{aligned}
& k_{1}=k g^{-3 / 2}=1 / r_{1}, \quad k_{2}=k g^{-1 / 2}=1 / r_{2} \\
& k=1 /(2 F)=1 / R, g=1+k^{2}\left(X_{0}^{2}+Y_{0}^{2}\right)
\end{aligned}
$$

In this paper, $\zeta_{\mathrm{APS}}$ is the APS (approximate parabolic surface) at $P_{0}$. The dimensionless parameter $g$ is related to the distance of $P_{0}$ from the center axis of the ideal parabolic surface. When $g$ is large, the distance from the center axis becomes large. For example, $g=1$ corresponds to the case of $P_{0}$ at the vertex, and $g=1.5$ and $g=2$ correspond to the cases where the distances from the center axis are $\sqrt{2} F$ and $2 F$, respectively. When $\xi$ and $\eta$ take small values, the APS approximately coincides with the ideal parabolic surface. Using the $P_{0}-x, y, \zeta$ system, the APS is expressed by

$$
\begin{gathered}
\zeta_{\mathrm{APS}}=(1 / 2)\left[\left(k_{1} \cos ^{2} \alpha+k_{2} \sin ^{2} \alpha\right) x^{2}+\left(k_{1} \sin ^{2} \alpha+k_{2} \cos ^{2} \alpha\right) y^{2}\right. \\
\left.+2 x y\left(k_{2}-k_{1}\right) \sin \alpha \cos \alpha\right]
\end{gathered}
$$

\section{Analysis}

Consider a membrane $\mathrm{M}_{\mathrm{R}}$ whose projection on the $\xi, \eta$ plane (or $x, y$ plane) is rectangular with its center point coinciding with $P_{0}$, as shown in Fig. 2(b). The length and width of the membrane are $2 a$ and $2 b$, respectively. The $x$ and $y$ axes are parallel to the sides. The edges are supported and subjected to constant tensile forces, $T_{x}$ and $T_{y}$. Assuming that the bending rigidity of the membrane can be neglected and the deflection is small, the following equation ${ }^{17-19)}$ is obtained for the deflection of the membrane:

$$
\partial^{2} w / \partial x^{2}+\kappa^{2} \partial^{2} w / \partial y^{2}=0
$$

where $\kappa$ is a constant and expressed by $\kappa=\sqrt{T_{y} / T_{x}}$, and $w$ is defined in $-a \leq x \leq a,-b \leq y \leq b$.

Assuming that the edges of the membrane are on an elliptic or hyperbolic paraboloidal surface, the deflection of the edges, $w_{\mathrm{B}}$, is expressed by 
Trans. JSASS Space Tech. Japan Vol. 4 (2006)

$$
\begin{aligned}
& w_{\mathrm{B}}=\left(l_{1} / 2\right) x^{* 2}+\left(l_{2} / 2\right) y^{* 2}+h \\
& =(1 / 2)\left[\left(l_{1} \cos ^{2} \beta+l_{2} \sin ^{2} \beta\right) x^{2}+\left(l_{1} \sin ^{2} \beta+l_{2} \cos ^{2} \beta\right) y^{2}\right. \\
& \left.+2 x y\left(l_{1}-l_{2}\right) \cos \beta \sin \beta\right]+h
\end{aligned}
$$

Equations (5a) and (5b) are equations of membrane deflection subjected to anisotropic tension and the boundary condition for the membrane $\mathrm{M}_{\mathrm{R}}$, respectively, in $-a \leq x \leq a,-b \leq y \leq b$.

By introducing a new parameter $y^{\prime}(=y / \kappa)$, Eq. (5a) becomes Laplace's equation:

$$
\partial^{2} w / \partial x^{2}+\partial^{2} w / \partial y^{\prime 2}=0
$$

where $w$ is defined in $-a \leq \mathrm{x} \leq a,-b / \kappa \leq y^{\prime} \leq b / \kappa$. Using the parameter $y^{\prime}$, Eq. $(5 \mathrm{~b})$ becomes

$$
\begin{aligned}
w_{\mathrm{B}}= & (1 / 2)\left[\left(l_{1} \cos ^{2} \beta+l_{2} \sin ^{2} \beta\right) x^{2}\right. \\
& +\kappa^{2}\left(l_{1} \sin ^{2} \beta+l_{2} \cos ^{2} \beta\right) y^{\prime 2} \\
& \left.+2 \kappa\left(l_{1}-l_{2}\right)(\cos \beta \sin \beta) x y^{\prime}\right]+h
\end{aligned}
$$

Equations (6a) and (6b) are equations of membrane deflection subjected to isotropic tension and the boundary condition, respectively, in $-a \leq x \leq a,-b / \kappa \leq y^{\prime} \leq b / \kappa$. When $w$ satisfies Eqs. (5a) and (5b), it also satisfies Eqs. (6a) and (6b), and vice versa.

In this paper, approximating the ideal parabolic surface with the APS, we define the rms error (i.e. surface error) of the membrane element based on the ideal parabolic surface using the following equation: $:^{15-20)}$

$$
\delta_{\text {rms }}=\left[\left(1 / A_{\mathrm{me}}\right) \iint_{S_{\mathrm{me}}}\left(w-\zeta_{\mathrm{APS}}\right)^{2} \mathrm{~d} s\right]^{1 / 2}
$$

where $S_{\mathrm{me}}$ is the projection region of the membrane element to the $\xi, \eta$ surface (or $x, y$ surface), $A_{\mathrm{me}}$ is the area of $S_{\mathrm{me}}$ and $\mathrm{d} s$ is the area element.

Here, the deflection of a membrane given by the following expression is considered:

$$
\begin{aligned}
& w=\left[l_{1}\left(\cos ^{2} \beta+\kappa^{2} \sin ^{2} \beta\right)+l_{2}\left(\sin ^{2} \beta+\kappa^{2} \cos ^{2} \beta\right)\right] \\
& \times a^{2}\left(16 / \pi^{3}\right) \Phi+w_{\mathrm{B}} \\
& \Phi=\sum_{n=1,3,5 \cdots}^{\infty}\left(1 / n^{3}\right)(-1)^{(n+3) / 2}\left[1-\frac{\cosh \left(n \pi y^{\prime} /(2 a)\right)}{\cosh \left(n \pi \lambda^{\prime} / 2\right)}\right] \cos \frac{n \pi x}{2 a} \\
& \lambda^{\prime}=\lambda / \kappa=(b / a) / \kappa, \lambda=b / a, y^{\prime}=y / \kappa
\end{aligned}
$$

Apparently, $\Phi$ takes the value of zero on the boundary of $x= \pm a$, $-b / \kappa \leq y^{\prime} \leq b / \kappa$ and $y^{\prime}= \pm b / \kappa,-a \leq x \leq a$, which means $w$ satisfies the boundary condition of Eq. (6b). Considering the following relation holds true, ${ }^{21)}$

$$
\partial^{2} \Phi / \partial x^{2}+\partial^{2} \Phi / \partial y^{\prime 2}=-\pi^{3} /\left(16 a^{2}\right),
$$

we can easily show that $w$ in Eq. (8) is the solution of Eq. (6a). Then, $w$ in Eq. (8) expresses the membrane deflection subjected to isotropic tension in $-a \leq x \leq a,-b / \kappa \leq y^{\prime} \leq b / \kappa$ with the boundary condition of Eq. (6b). Consequently, w in Eq. (8) also expresses the membrane deflection subjected to anisotropic tensions $T_{x}$ and $T_{y}$ in $-a \leq x \leq a,-b \leq y \leq b$ with the boundary condition of Eq. (5b).

From Eqs. (4) , (5b) and (8), the following relation is obtained:

$$
\begin{aligned}
\frac{F\left(w-\zeta_{\mathrm{APS}}\right)}{4 a b}= & D_{0} \Phi+H \\
& +\frac{1}{2}\left[A_{0}\left(\frac{x}{a}\right)^{2}+B_{0}\left(\frac{y}{a}\right)^{2}+2 C_{0}\left(\frac{x}{a}\right)\left(\frac{y}{a}\right)\right]
\end{aligned}
$$

where

$$
\begin{aligned}
& D_{0}=\left(16 / \pi^{3}\right)\left[L_{1}\left(\cos ^{2} \beta+\kappa^{2} \sin ^{2} \beta\right)\right. \\
& \left.\quad+L_{2}\left(\sin ^{2} \beta+\kappa^{2} \cos ^{2} \beta\right)\right] \\
& A_{0}=\left(L_{1} \cos ^{2} \beta+L_{2} \sin ^{2} \beta\right)-\left(K_{1} \cos ^{2} \alpha+K_{2} \sin ^{2} \alpha\right) \\
& B_{0}=\left(L_{1} \sin ^{2} \beta+L_{2} \cos ^{2} \beta\right)-\left(K_{1} \sin ^{2} \alpha+K_{2} \cos ^{2} \alpha\right) \\
& C_{0}=\left(L_{1}-L_{2}\right) \cos \beta \sin \beta-\left(K_{2}-K_{1}\right) \sin \alpha \cos \alpha \\
& L_{1}=F l_{1} /(4 \lambda), L_{2}=F l_{2} /(4 \lambda) \\
& K_{1}=F k_{1} /(4 \lambda), K_{2}=F k_{2} /(4 \lambda) \\
& H=F h /(4 a b)
\end{aligned}
$$

Substituting Eqs. (10) and (11) into Eq. (7) produces

$$
\begin{aligned}
F^{2} \delta_{\text {rms }}{ }^{2} /\left(16 a^{2} b^{2}\right)= & D_{0}{ }^{2} L_{a}+D_{0} A_{0} L_{b a}+D_{0} B_{0} L_{b b} \kappa^{2}+2 D_{0} H L_{c} \\
& +A_{0}{ }^{2} / 20+B_{0}{ }^{2} \lambda^{4} / 20+C_{0}{ }^{2} \lambda^{2} / 9 \\
& +A_{0} B_{0} \lambda^{2} / 18+H\left(A_{0}+B_{0} \lambda^{2}\right) / 3+H^{2}
\end{aligned}
$$

where $L_{a}, L_{b a}, L_{b b}$ and $L_{c}$ are functions of $\lambda^{\prime}$, and expressed by

$$
\begin{aligned}
L_{a} & =\left(1 / A_{\mathrm{me}}\right) \iint_{S_{\mathrm{me}}} \Phi^{2} \mathrm{~d} s \\
& =\frac{1}{4 \lambda^{\prime}} \sum_{n=1,3,5}^{\infty} \ldots \frac{1}{n^{6}}\left[3 \lambda^{\prime}-\frac{6}{n \pi} \tanh \left(n \pi \lambda^{\prime} / 2\right)-\lambda^{\prime} \tanh ^{2}\left(n \pi \lambda^{\prime} / 2\right)\right] \\
L_{b a} & =\frac{1}{a^{2} A_{\mathrm{me}}} \iint_{S_{\mathrm{me}}} \Phi x^{2} \mathrm{~d} s \\
& =\frac{1}{4 \lambda^{\prime}} \sum_{n=1,3,5}^{\infty} \frac{16}{\pi^{2}} \frac{(n \pi / 2)^{2}-2}{n^{6} \pi}\left[2 \lambda^{\prime}-\frac{4}{n \pi} \tanh \left(n \pi \lambda^{\prime} / 2\right)\right] \\
L_{b b} & =\frac{1}{a^{2} A_{\mathrm{me}}} \iint_{S_{\mathrm{me}}} \Phi y^{2} \mathrm{~d} s \\
= & \frac{1}{4 \lambda^{\prime}} \sum_{n=1,3,5}^{\infty} \ldots \frac{16}{n^{5} \pi^{2}}\left[\frac{n \pi}{6}\left(\lambda^{\prime}\right)^{3}+\frac{4 \lambda^{\prime}}{n \pi}-\left\{\left(\lambda^{\prime}\right)^{2}+2\left(\frac{2}{n \pi}\right)^{2}\right\} \tanh \frac{n \pi \lambda^{\prime}}{2}\right] \\
L_{c} & =\left(1 / A_{\mathrm{me}}\right) \iint_{S_{\mathrm{me}}} \Phi \mathrm{d} s \\
& =\frac{1}{4 \lambda^{\prime}} \sum_{n=1,3,5}^{\infty} \frac{4}{n^{4} \pi}\left[2 \lambda^{\prime}-\frac{4}{n \pi} \tanh \left(n \pi \lambda^{\prime} / 2\right)\right]
\end{aligned}
$$

When $\beta=-\alpha, l_{1}=k_{1}$ and $l_{1}=k_{2}$, the formulations of membrane deflection (Eq. (10)) and surface error (Eq. (12)) reduce to those of Ref. 19).

In the following, optimization of the surface error of a membrane subjected to anisotropic tension is carried out by minimizing the rms error with respect to parameters, $L_{1}, L_{2}, H$ and $\beta$ (or $l_{1}, l_{2}, h$ and $\beta$ ) that determine the normal deflection of the edges of the membrane. Analytical formulations for the surface error and the deflection of three kinds of optimized rectangular membranes subjected to anisotropic tension are shown. One is an equalcurvature-edge-optimum membrane, where all edges have equal curvatures $\left(L_{1}=L_{2}=L_{\mathrm{e}}\right)$ and the surface error is minimized with respect to $L_{\mathrm{e}}$ and $H$. The others are; an optimum-2 membrane, whose surface error is optimized with respect to $L_{1}, L_{2}, H$ and $\beta$; and an optimum-3 membrane, where $\beta$ is specified as zero and the surface error is minimized with respect to $L_{1}, L_{2}$ and $H$. 
S. KATO: Effects of Tension and Edge Deflection upon the Surface Accuracy of a Rectangular Membrane of a Space Reflector

\subsection{Equal-curvature-edge-optimum membrane}

The equal-curvature-edge-optimum membrane is obtained by specifying equal curvatures on all edges (i.e., $L_{1}=L_{2}=L_{\mathrm{e}}$ ) and minimizing the surface error with respect to $L_{\mathrm{e}}$ and the normal translation $H$.

Substituting $L_{1}=L_{2}=L_{\mathrm{e}}$ into Eqs. (12) and (11) yields

$$
\begin{aligned}
F^{2} \delta_{\text {rms }}{ }^{2} /\left(16 a^{2} b^{2}\right)= & D_{\mathrm{e}}{ }^{2} L_{a}+D_{\mathrm{e}} A_{\mathrm{e}} L_{b a}+D_{\mathrm{e}} B_{\mathrm{e}} L_{b b} \kappa^{2}+2 D_{\mathrm{e}} H L_{\mathrm{e}} \\
& +A_{\mathrm{e}}{ }^{2} / 20+B_{\mathrm{e}}{ }^{2} \lambda^{4} / 20+C_{\mathrm{e}}{ }^{2} \lambda^{2} / 9 \\
& +A_{\mathrm{e}} B_{\mathrm{e}} \lambda^{2} / 18+H\left(A_{\mathrm{e}}+B_{\mathrm{e}} \lambda^{2}\right) / 3+H^{2}
\end{aligned}
$$

where

$$
\begin{array}{ll}
D_{\mathrm{e}}=L_{\mathrm{e}}\left(1+\kappa^{2}\right)\left(16 / \pi^{3}\right) \\
A_{\mathrm{e}}=L_{\mathrm{e}}+A_{1}, & A_{1}=-\left(K_{1} \cos ^{2} \alpha+K_{2} \sin ^{2} \alpha\right) \\
B_{\mathrm{e}}=L_{\mathrm{e}}+B_{1}, & B_{1}=-\left(K_{1} \sin ^{2} \alpha+K_{2} \cos ^{2} \alpha\right) \\
C_{\mathrm{e}}=C_{1}, & C_{1}=-\left(K_{2}-K_{1}\right) \sin \alpha \cos \alpha \\
L_{\mathrm{e}}=F l_{\mathrm{e}} /(4 \lambda) &
\end{array}
$$

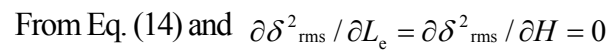

$$
\left(\begin{array}{ll}
c_{11} & c_{12} \\
c_{21} & c_{22}
\end{array}\right)\left(\begin{array}{l}
L_{\mathrm{e}} \\
H
\end{array}\right)=\left(\begin{array}{l}
c_{10} \\
c_{20}
\end{array}\right)
$$

where

$$
\begin{aligned}
c_{11}= & 2\left(1+\kappa^{2}\right)^{2}\left(16 / \pi^{3}\right)^{2} L_{a} \\
& +2\left(1+\kappa^{2}\right)\left(16 / \pi^{3}\right)\left(L_{b a}+\kappa^{2} L_{b b}\right) \\
& +1 / 10+\lambda^{2} / 9+\lambda^{4} / 10 \\
c_{12}= & 2\left(1+\kappa^{2}\right)\left(16 / \pi^{3}\right) L_{c}+\left(1+\lambda^{2}\right) / 3 \\
c_{10}= & -\left(1+\kappa^{2}\right)\left(16 / \pi^{3}\right)\left(A_{1} L_{b a}+\kappa^{2} B_{1} L_{b b}\right) \\
& -A_{1} / 10-B_{1} \lambda^{4} / 10-\left(A_{1}+B_{1}\right) \lambda^{2} / 18 \\
c_{21}= & 2\left(1+\kappa^{2}\right)\left(16 / \pi^{3}\right) L_{c}+\left(1+\lambda^{2}\right) / 3 \\
c_{22}= & 2 \\
c_{20}= & -\left(A_{1}+B_{1} \lambda^{2}\right) / 3
\end{aligned}
$$

Solving Eq. (16) yields

$$
\begin{aligned}
& L_{\mathrm{e}}=\left(c_{10} c_{22}-c_{20} c_{12}\right) /\left(c_{11} c_{22}-c_{21} c_{12}\right) \\
& H=\left(c_{10} c_{21}-c_{20} c_{11}\right) /\left(c_{12} c_{21}-c_{22} c_{11}\right)
\end{aligned}
$$

Substituting $L_{1}=L_{2}=L_{\mathrm{e}}$ and $H$ given from Eq. (18) into Eqs. (14), (15), (10) and (11), and using Eqs. (4) and (8), we obtain the surface error $\delta_{1}\left[=F \delta_{\text {rms }} /(4 a b)\right]$ and the deflection of an equalcurvature-edge-optimum membrane.

\subsection{Optimum-2 membrane and optimum-3 membrane}

A two-curvature membrane is obtained by minimizing the surface error with respect to $L_{1}, L_{2}$ and $H$.

We require

$$
\partial \delta_{\text {rms }}^{2} / \partial L_{1}=\delta_{\text {rms }}^{2} / \partial L_{2}=\partial \delta_{\text {rms }}^{2} / \partial H=0
$$

which leads to the following equations determining the optimum values of $L_{1}, L_{2}$ and $H$ :

$$
\begin{aligned}
& \left(\begin{array}{lll}
e_{11} & e_{12} & e_{13} \\
e_{21} & e_{22} & e_{23} \\
e_{31} & e_{32} & e_{33}
\end{array}\right)\left(\begin{array}{l}
L_{1} \\
L_{2} \\
H
\end{array}\right)=\left(\begin{array}{l}
e_{1} \\
e_{2} \\
e_{3}
\end{array}\right) \\
& e_{11}=2\left(16 / \pi^{3}\right)^{2}\left(\cos ^{2} \beta+\kappa^{2} \sin ^{2} \beta\right)^{2} L_{a} \\
& +2\left(16 / \pi^{3}\right)\left(\cos ^{2} \beta+\kappa^{2} \sin ^{2} \beta\right)\left(L_{b a} \cos ^{2} \beta+\kappa^{2} L_{b b} \sin ^{2} \beta\right) \\
& +(1 / 10) \cos ^{4} \beta+\left(\lambda^{4} / 10\right) \sin ^{4} \beta+\left(\lambda^{2} / 3\right) \cos ^{2} \beta \sin ^{2} \beta \\
& e_{12}=2\left(16 / \pi^{3}\right)^{2}\left(\sin ^{2} \beta+\kappa^{2} \cos ^{2} \beta\right)\left(\cos ^{2} \beta+\kappa^{2} \sin ^{2} \beta\right) L_{a} \\
& +\left(16 / \pi^{3}\right)\left[\left(\cos ^{2} \beta+\kappa^{2} \sin ^{2} \beta\right) \sin ^{2} \beta\right. \\
& \left.+\left(\sin ^{2} \beta+\kappa^{2} \cos ^{2} \beta\right) \cos ^{2} \beta\right] L_{b a} \\
& +\left(16 / \pi^{3}\right)\left[\left(\cos ^{2} \beta+\kappa^{2} \sin ^{2} \beta\right) \cos ^{2} \beta\right. \\
& \left.+\left(\sin ^{2} \beta+\kappa^{2} \cos ^{2} \beta\right) \sin ^{2} \beta\right] L_{b b} \kappa^{2} \\
& +(1 / 10)\left(1+\lambda^{4}\right) \sin ^{2} \beta \cos ^{2} \beta-(2 / 9) \lambda^{2} \sin ^{2} \beta \cos ^{2} \beta \\
& +\left(\lambda^{2} / 18\right)\left(\sin ^{4} \beta+\cos ^{4} \beta\right) \\
& e_{13}=2\left(16 / \pi^{3}\right)\left(\cos ^{2} \beta+\kappa^{2} \sin ^{2} \beta\right) L_{c}+\left(\cos ^{2} \beta+\lambda^{2} \sin ^{2} \beta\right) / 3 \\
& e_{1}=-\left(16 / \pi^{3}\right)\left(\cos ^{2} \beta+\kappa^{2} \sin ^{2} \beta\right)\left(A_{1} L_{b a}+\kappa^{2} B_{1} L_{b b}\right) \\
& -(1 / 10) A_{1} \cos ^{2} \beta-\left(\lambda^{4} / 10\right) B_{1} \sin ^{2} \beta-(2 / 9) \lambda^{2} C_{1} \sin \beta \cos \beta \\
& -\left(\lambda^{2} / 18\right)\left(A_{1} \sin ^{2} \beta+B_{1} \cos ^{2} \beta\right) \\
& e_{21}=e_{12} \\
& e_{22}=2\left(16 / \pi^{3}\right)^{2}\left(\sin ^{2} \beta+\kappa^{2} \cos ^{2} \beta\right)^{2} L_{a} \\
& +2\left(16 / \pi^{3}\right)\left(\sin ^{2} \beta+\kappa^{2} \cos ^{2} \beta\right)\left(L_{b a} \sin ^{2} \beta+\kappa^{2} L_{b b} \cos ^{2} \beta\right) \\
& +(1 / 10) \sin ^{4} \beta+\left(\lambda^{4} / 10\right) \cos ^{4} \beta+\left(\lambda^{2} / 3\right) \cos ^{2} \beta \sin ^{2} \beta \\
& e_{23}=2\left(16 / \pi^{3}\right)\left(\sin ^{2} \beta+\kappa^{2} \cos ^{2} \beta\right) L_{c}+\left(\sin ^{2} \beta+\lambda^{2} \cos ^{2} \beta\right) / 3 \\
& e_{2}=-\left(16 / \pi^{3}\right)\left(\sin ^{2} \beta+\kappa^{2} \cos ^{2} \beta\right)\left(A_{1} L_{b a}+\kappa^{2} B_{1} L_{b b}\right) \\
& -(1 / 10) A_{1} \sin ^{2} \beta-\left(\lambda^{4} / 10\right) B_{1} \cos ^{2} \beta+(2 / 9) \lambda^{2} C_{1} \sin \beta \cos \beta \\
& -\left(\lambda^{2} / 18\right)\left(A_{1} \cos ^{2} \beta+B_{1} \sin ^{2} \beta\right) \\
& e_{31}=e_{13}, \quad e_{32}=e_{23}, \quad e_{33}=2 \\
& e_{3}=-\left(A_{1}+B_{1} \lambda^{2}\right) / 3
\end{aligned}
$$

Substituting the solutions of Eq. (20), $L_{1}, L_{2}$ and $H$ into Eqs. (11) and (12), and using Eqs. (4), (8) and (10), we obtain a two-curvature membrane whose surface error $F \delta_{\text {rms }} /(4 a b)$ and the membrane deflection $w$ depend on the parameters of $\alpha, \lambda, \kappa, \beta$ and $g$. Minimizing the surface error of a two-curvature membrane with respect to $\beta$, we obtain an optimum- 2 membrane. When $\beta$ of a two-curvature membrane has a value of zero $(\beta=0)$, the two-curvature membrane becomes an optimum- 3 membrane. Each edge of the optimum-3 membrane has the same curvature as the opposite edge, where $L_{1}$ is specified on $\mathrm{AB}$ and $\mathrm{CD}$, and $L_{2}$ is specified on $\mathrm{AD}$ and $\mathrm{BC}$.

\section{Results and Discussion}

Figures 3 and 4(a)-(d) show the effects of $\kappa$ and $\lambda$ upon the surface errors $\left(\delta_{1}, \delta_{\text {2opt }}\right.$ and $\left.\delta_{3}\right)$ for several combinations of $g$ and $\alpha$. The surface error $\delta_{\text {copt }}$ of a coincident-optimum-edge membrane ${ }^{19)}$ is also shown for comparison. [A coincidentoptimum-edge membrane is obtained by minimizing the rms error using the normal translation of a coincident-edge membrane whose edges coincide with the approximate parabolic surface of the local point where the membrane is placed. The surface error $\delta_{\text {copt }}$ is used for the purpose of comparison, and is about $0.4-0.5$ of $\delta_{\mathrm{c}}$, the surface error of a coincident-edge membrane of Ref. 19).]

Figure. 3 corresponds to the case in which the membrane is 


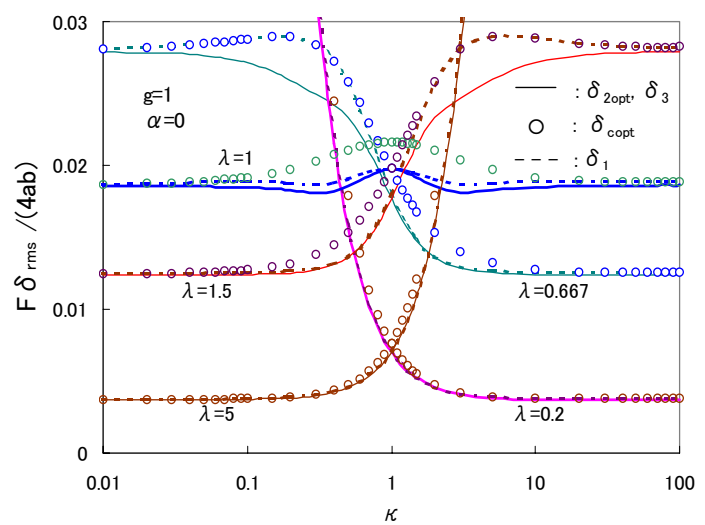

Fig. 3. Effects of $\kappa$ and $\lambda$ upon the surface errors $(g=1, \alpha=0)$.

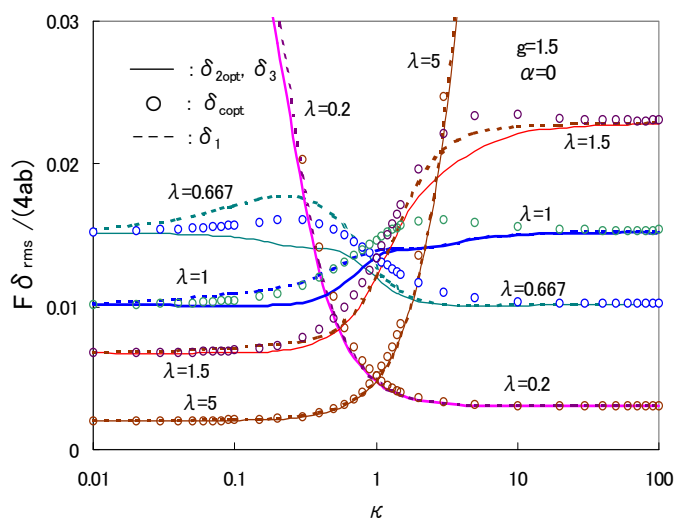

Fig. 4(a). Effects of $\kappa$ and $\lambda$ upon the surface errors $(g=1.5, \alpha=0)$.

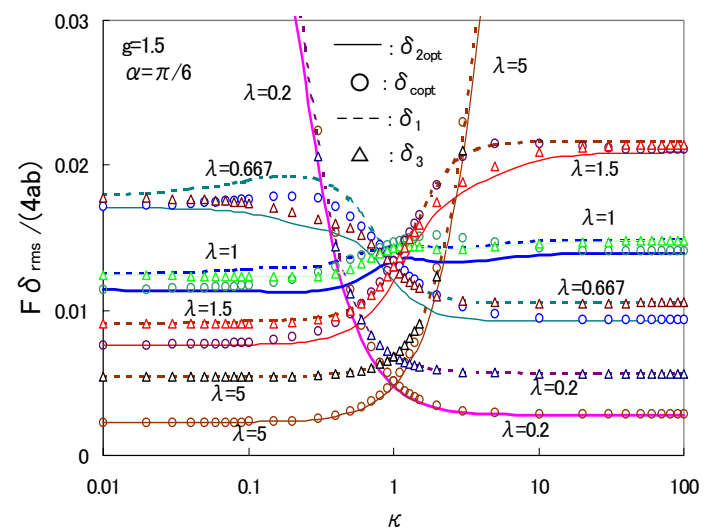

Fig. 4(b). Effects of $\kappa$ and $\lambda$ upon the surface errors $(g=1.5, \alpha=\pi / 6)$.

located at the vertex of the base parabolic surface of a reflector $(g=1$, $\alpha=0$ ). Apparently, when $g=1$ the surface errors do not depend on $\alpha$. From Fig. 3, it is seen that $\delta_{2 \text { opt }}$ and $\delta_{3}$ are equal and the following relation is obtained for any $\lambda$ and $\kappa$ :

$$
\delta_{\text {copt }}>\delta_{1} \geq \delta_{2 \mathrm{opt}}=\delta_{3}
$$

When $\lambda=1$, the surface errors $\delta_{1}, \delta_{\text {2opt }}, \delta_{3}$ and $\delta_{\text {copt }}$ are

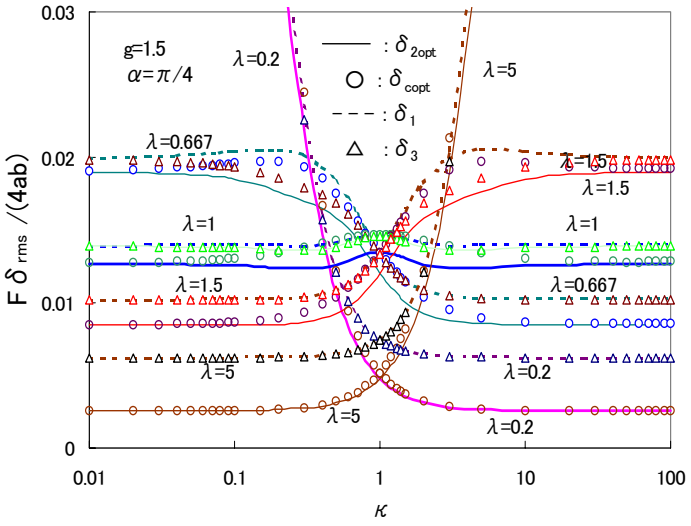

Fig. 4(c). Effects of $\kappa$ and $\lambda$ upon the surface errors $(g=1.5, \alpha=\pi / 4)$.

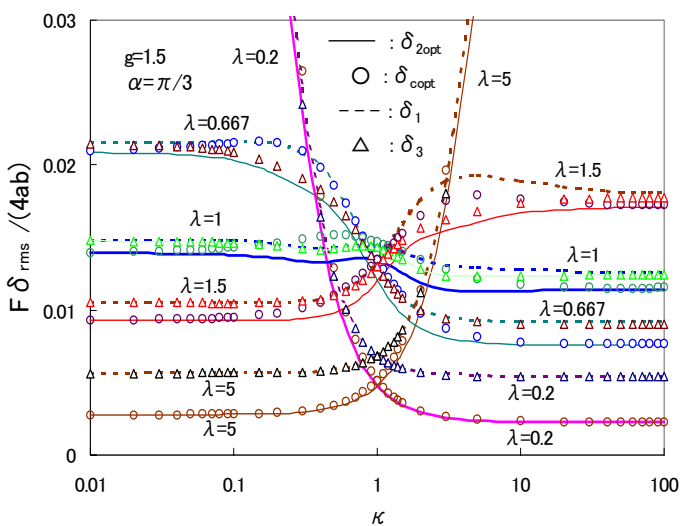

Fig. 4(d). Effects of $\kappa$ and $\lambda$ upon the surface errors $(g=1.5, \alpha=\pi / 3)$.

symmetric with respect to the line of $\kappa=1$ in the logarithmic axis of $\kappa$. Each of the surface errors has a relative maximum at $\kappa=1$. Whereas $\delta_{\text {copt }}$ decreases monotonically as $\kappa$ increases or decreases from 1 , each of $\delta_{1}, \delta_{2 \text { opt }}$ and $\delta_{3}$ has a relative minimum at the point of $\kappa \cong 3$ or $\kappa \cong 0.3$. When $\lambda$ is 1 , except for $\delta_{\text {copt }}$, the difference between $\delta_{1}$ and $\delta_{\text {2opt }}\left(=\delta_{3}\right)$ is small. When $\lambda$ is 1 and $\kappa$ is in the neighborhood of $1, \delta_{1}, \delta_{2 \text { opt }}$ and $\delta_{3}$ are about $10 \%$ less than $\delta_{\text {copt }}$. These surface errors approach each other as $\kappa$ increases or decreases. The dependency of the surface errors on $\kappa$ with $\lambda=1$ is small compared with other slender rectangular membranes.

Figure 4(a) shows the relation between $\kappa, \lambda$ and the surface errors for $g=1.5$ with $\alpha=0$. It is seen that $\delta_{2 \text { opt }}$ and $\delta_{3}$ are equal, which is the same as in the case of $g=1$. In Fig. 4(a), the following relation is obtained for any $\lambda$ and $\kappa$ :

$$
\delta_{\text {copt }}, \delta_{1}>\delta_{2 \text { opt }}=\delta_{3}
$$

When $\lambda=1$, each surface error decreases as $\kappa$ decreases in the neighborhood of $\kappa=1$.

Figures 4(b)-(d) show the effects of $\kappa$ and $\lambda$ upon the surface errors for $g=1.5$ with $\alpha=\pi / 6, \pi / 4$ and $\pi / 3$. The overall behavior of these surface errors is roughly similar to that of Fig. 3 or Fig. 4(a). These surface errors are not equal at $\alpha \neq 0$ in Figs. 4(b)-(d), whereas $\delta_{2 \text { opt }}$ and $\delta_{3}$ are equal at $\alpha=0$ in Figs. 3 and 4(a). The following relation is obtained for any $\lambda$ and $\kappa$ : 
S. KATO: Effects of Tension and Edge Deflection upon the Surface Accuracy of a Rectangular Membrane of a Space Reflector

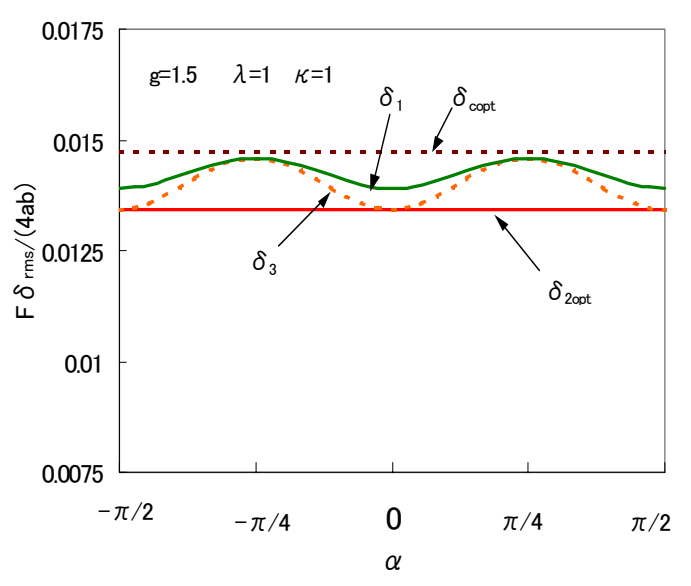

Fig. 5(a). Effect of $\alpha$ upon the surface errors $(g=1.5, \lambda=1)$.

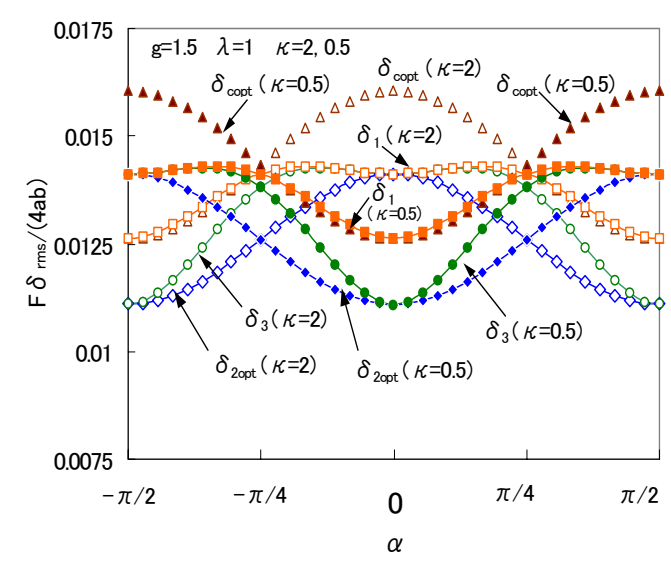

Fig. 5(b). Effects of $\alpha$ upon the surface errors $(g=1.5, \lambda=1)$.

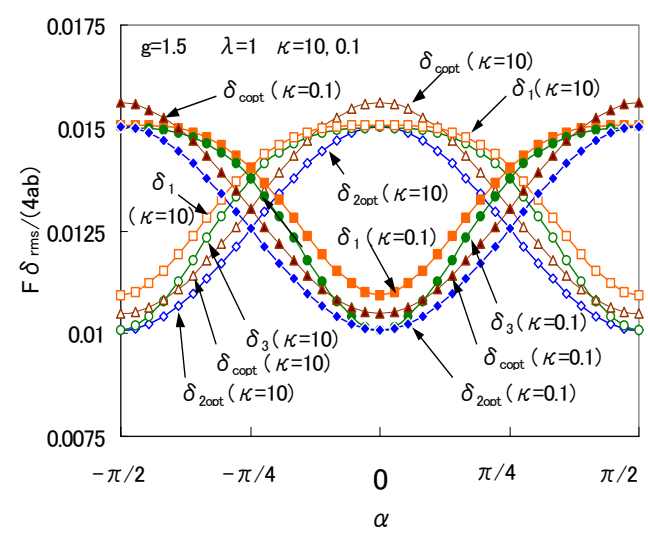

Fig. 5(c). Effects of $\alpha$ upon the surface errors $(g=1.5, \lambda=1)$.

$$
\begin{gathered}
\delta_{1}>\delta_{3}>\delta_{2 \mathrm{opt}} \\
\delta_{\mathrm{copt}}>\delta_{2 \mathrm{opt}}
\end{gathered}
$$

In Figs. 4(b)-(d), the surface error $\delta_{2 \text { opt }}$ is about $0-15 \%$ less than $\delta_{\text {copt }}$. When $\lambda=1$ and $\alpha=\pi / 4$ (Fig. 4(c)), each surface error is symmetrical with respect to the line of $\kappa=1$ in the logarithmic axis

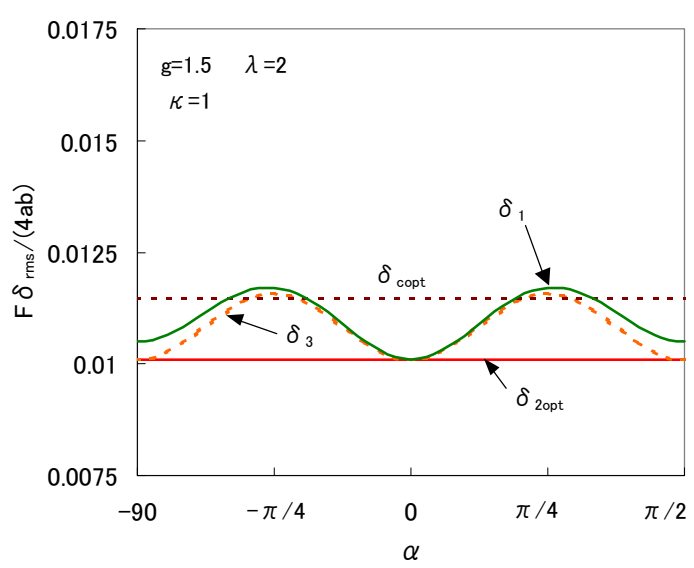

Fig. 6(a). Effects of $\alpha$ upon the surface errors $(g=1.5, \lambda=2)$.

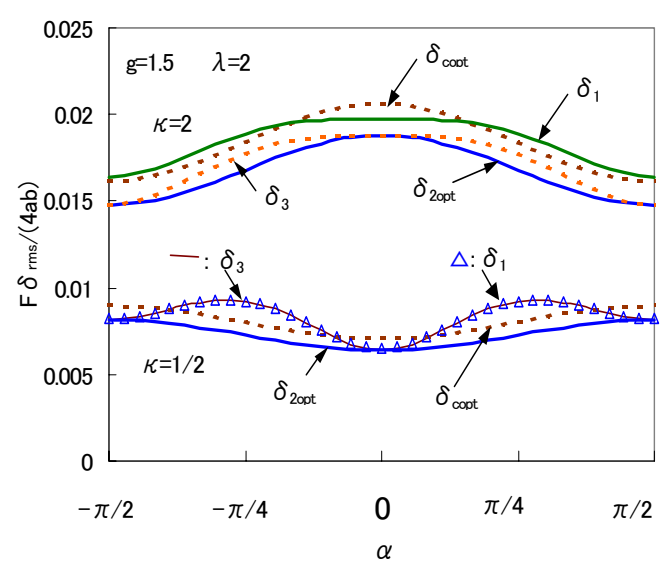

Fig. 6(b). Effects of $\alpha$ upon the surface errors $(g=1.5, \lambda=2)$.

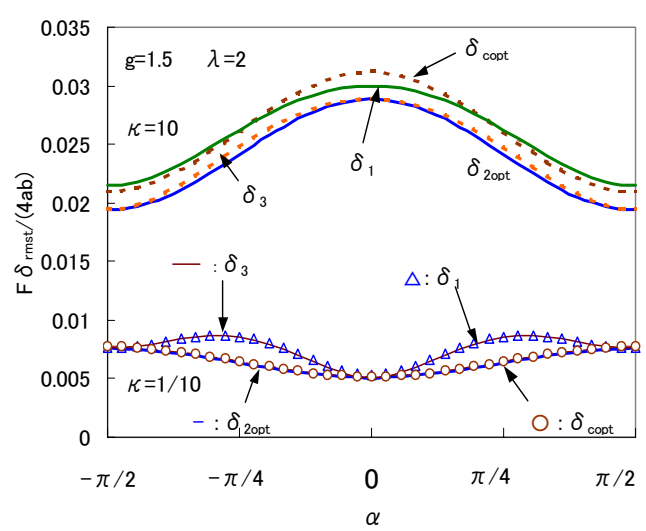

Fig. 6(c). Effects of $\alpha$ upon the surface errors $(g=1.5, \lambda=2)$.

of $\kappa$.

From Figs. 3 and 4(a)-(d), the tendencies of surface errors with respect to $\kappa$ and $\lambda$ are as follows:

(1) When $\lambda$ is large enough compared to 1 , each surface error with the values of $\kappa(<1)$ and $\lambda$ is smaller than that for $1 / \kappa$ and $\lambda$. When $\lambda$ is small enough compared to 1 , each surface error with the values of $\kappa(>1)$ and $\lambda$ is smaller than that for $1 / \kappa$ and $\lambda$. 
(2) When $\lambda$ is large enough compared to 1 , each surface error decreases as $\kappa$ decreases. When $\lambda$ is small enough compared to 1 , each surface error decreases as $\kappa$ increases. These tendencies are remarkable when $\kappa$ is near 1 .

(3) With the value of $\alpha=0$ (Figs. 3, 4(a)), $\delta_{2 \text { opt }}$ is equal to $\delta_{3}$, and when $\lambda$ is large enough and $\kappa$ is small enough or $\lambda$ is small enough and $\kappa$ is large enough compared to 1 , $\delta_{\text {2opt }}\left(=\delta_{3}\right)$ is approximately equal to $\delta_{1}$ and $\delta_{\text {copt }}$. With the value of $\alpha \neq 0$ [Figs. 4(b)-(d)], when $\lambda$ is large enough and $\kappa$ is small enough or $\lambda$ is small enough and $\kappa$ is large enough compared to $1, \delta_{\text {opt }}\left(\cong \delta_{\text {copt }}\right)$ is smaller than $\delta_{3}\left(\cong \delta_{1}\right)$, which is different from the case of $\alpha=0$. The ratio of $\delta_{2 \text { opt }} / \delta_{3}$ is about 0.5 for $\alpha=\pi / 6, \pi / 4$, $\pi / 3$ and $\lambda=5,1 / 5$.

(4) When $\lambda$ is large enough and $\kappa$ is small enough or $\lambda$ is small enough and $\kappa$ is large enough compared to 1 , the surface errors take approximately constant values as $\kappa$ increases or decreases away from 1 .

(5) When $\lambda$ is large enough and $\kappa$ is small enough or $\lambda$ is small enough and $\kappa$ is large enough compared to 1 , the surface errors decrease as $\lambda$ increases in the range of $\kappa<1$ or as $\lambda$ decreases in the range of $\kappa>1$.

(6) In the neighborhood of $\kappa=1$ and except in the neighborhood of $\lambda=1$, the surface errors decrease as $\lambda$ increases or decreases from 1 .

(7) The surface error $\delta_{2 \text { opt }}$ is about $0-15 \%$ less than $\delta_{\text {copt }}$.

From (1), (2) and (5), the following tendency is obtained: The surface error of a slender membrane decreases if the longer side is loaded with a higher tension, and the higher the ratio of tension (ratio of higher tension to lower tension) is and as the slenderness of the membrane increases, the smaller the rms error is.

From Figs. 3 and 4(a)-(d), it is seen that with arbitrary values of $\alpha, g$ and $\lambda$, the appropriate tension ratio parameter $\kappa$ can reduce the surface error of each of the optimized rectangular membranes as compared to isotropic tension, which corresponds to the case of $\kappa=1$.

As mentioned above, when $g=1$, the surface errors do not depend on $\alpha$. The relations between $\alpha$ and the surface errors with $g=1.5$ and $\kappa=1,2,1 / 2,10$ and $1 / 10$ are shown in Figs. 5(a)-(c) for $\lambda=1$ and in Figs. 6(a)-(c) for $\lambda=2$. The surface error $\delta_{\text {copt }}$ of Ref. 19) is also shown for comparison. It is easily shown that the parallel translation of a curve for $\lambda$ and $\kappa$ with the amount of $\alpha=\pi / 2$ along the $\alpha$ axis gives the curve for $1 / \lambda$ and $1 / \kappa$. From Figs. 5(a) and 6(a), it is seen that when $g=1.5, \lambda=1, \kappa=1$, or $g=1.5$, $\lambda=2, \kappa=1$, the surface errors $\delta_{\text {2opt }}$ and $\delta_{\text {copt }}$ are constant. $\delta_{1}$ and $\delta_{3}$ have relative minima at $\alpha=0$ and relative maxima at $\alpha=$ $\pm \pi / 4$. From Figs. 5(b)-(c) and 6(b)-(c), when $\lambda$ is 1 or 2 and $\kappa$ is large enough compared to the value of 1 , the surface errors have relative maxima (or maximum values) at $\alpha=0$ and relative minima (or minimum values) at $\alpha= \pm \pi / 2$. It is also seen that when $\lambda$ is 1 or 2 , and $\kappa$ is smaller than $1, \delta_{\text {2opt }}$ and $\delta_{\text {copt }}$ have relative minima (or minimum values) at $\alpha=0$ and relative maxima (or maximum values) at $\alpha= \pm \pi / 2$; and $\delta_{1}$ and $\delta_{3}$ have relative minima (or minimum values) at $\alpha=0$, and maximum values at $\alpha \cong \pm \pi / 4$ or $\alpha= \pm \pi / 2$, according to the values of $\kappa$ and $\lambda$.

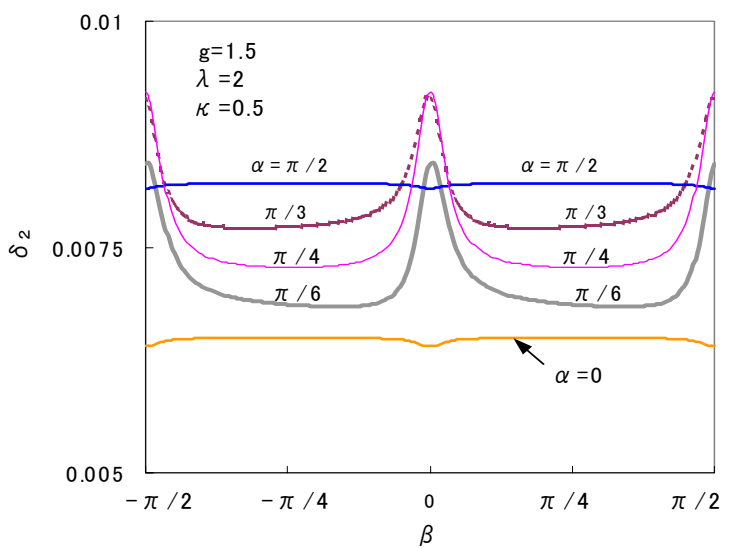

Fig. 7(a). Effects of $\beta$ upon the surface error $\delta_{2}$.

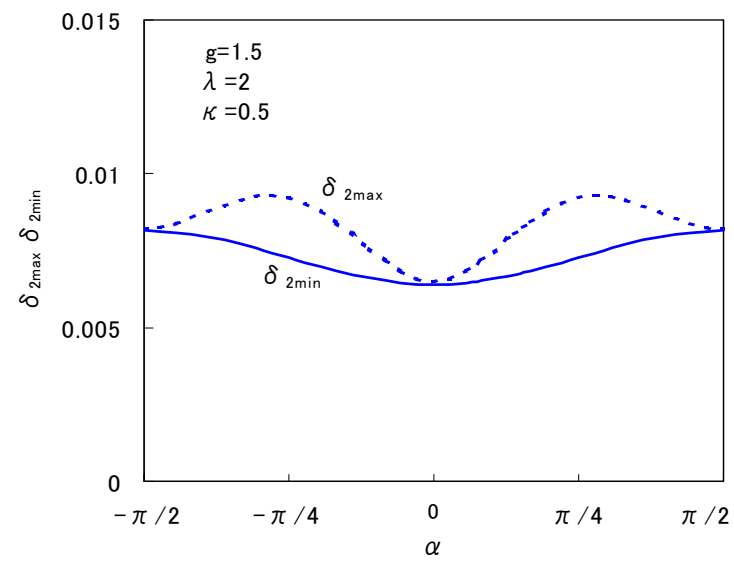

Fig. 7(b). Effects of $\alpha$ upon $\delta_{2 \max }$ and $\delta_{2 \min }$.

From Figs. 5(a)-(c), 6(a)-(c) and the above discussion, the following tendency is obtained:

The surface error of each of the optimized membranes has a relative maximum or a relative minimum when the side is parallel to the direction of principal curvature at the point where the membrane is located. For a slender membrane, the minimum surface error is obtained when the longer side is parallel to the $\eta$ axis and the longer side is loaded with higher tension. For this slender membrane with a minimum surface error, each surface error has a minimum value at $\alpha=0 ; \delta_{\text {2opt }}$ and $\delta_{\text {copt }}$ have maximum values at $\alpha= \pm \pi / 2$; and $\delta_{1}$ and $\delta_{\text {copt }}$ have maximum values at $\alpha \cong \pm \pi / 4$.

The surface error of a two-curvature membrane, which is given by minimizing the surface error with respect to $l_{1}, l_{2}$ and $h$ (or $L_{1}, L_{2}$, and $H$ ), depends on $\beta$. Figure. 7(a) shows the relation between $\beta$ and the surface error of a two-curvature membrane $\delta_{2}$ for several values of $\alpha$. With each value of $\alpha$, the surface error has a minimum and a maximum, where the minimum value corresponds to that of an optimum-2 membrane $\delta_{2 \text { opt }}$. Figure. 7(b) shows the dependency of maximum and minimum values of a two-curvature membrane, $\delta_{2 \max }$ and $\delta_{2 \min }$, upon $\alpha$, where $\delta_{2 \min }=\delta_{2 \text { opt }}$. 


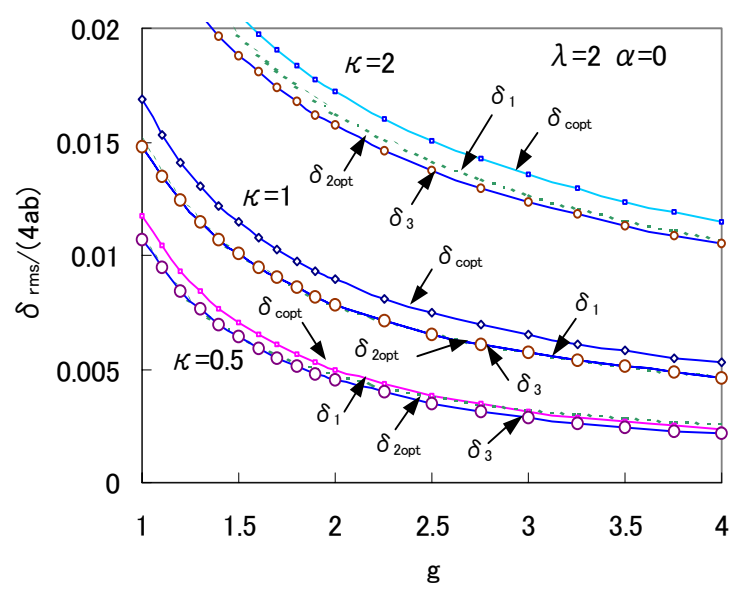

Fig. 8(a). Effects of $g$ upon the surface errors.

Figures. 8(a)-(b) show some examples of the effects of $g$ and $\kappa$ upon the surface errors including $\delta_{\text {cont }}$ from Ref. 19). From these figures, it is seen that, as $g$ increases, all surface errors become small. From Fig. 8(a), as $g$ increases, and with the value of $\alpha=0$, the differences of surface errors for each $\kappa$ do not change very much. However, in Fig. 8(b), with the value of $\alpha=\pi / 6$, differences between $\left(\delta_{1}, \delta_{3}\right)$ and $\left(\delta_{2 \text { opt }}, \delta_{\text {copt }}\right)$ become large as $g$ increases .

\section{Conclusions}

Considering a rectangular membrane that constitutes the reflector surface, such as that shown in Fig. 1, various parameters such as the aspect ratio $\lambda$, rotation angle $\alpha$, location of the membrane in the reflector $g$, tension ratio parameter $\kappa$ and edge deflection influence the surface error of the membrane. For the design of a reflector structure, it is very important to understand the relation between the surface error of the membrane and these parameters in order to obtain a highly accurate surface. The surface errors of a rectangular membrane for three kinds of optimized edge deflections with various parameters such as $\kappa, \lambda, \alpha$ and $g$ in a reflector are analyzed and evaluated based on an analytical solution of the linear membrane equation. These surface errors are also compared with that of a coincident-optimum-edge membrane studied in Ref. 19). Using the results of this paper, the design of a rectangular membrane subjected to anisotropic tension with high surface accuracy in a reflector, as shown in Fig. 1, would be possible.

Some details of the conclusions are as follows:

(1) The general analytical formulation for the surface error of a rectangular membrane subjected to anisotropic tension with parameters such as $\lambda, \alpha, \beta, g, L_{1}$ and $L_{2}$ is obtained based on the linear membrane equation. Substitution of $L_{1}=K_{1}, L_{2}=K_{2}$ and $\beta=-\alpha$ yields the formulation of Ref. 19). The analytical formulation in this paper would be useful for the design of a rectangular membrane subjected to anisotropic tension in a reflector.

(2) With arbitrary values of $\alpha, g$ and $\lambda$, the appropriate tension ratio parameter $\kappa$ can reduce the surface error of each of the optimized rectangular membranes as compared to the isotropic tension (isotropic tension corresponds to $\kappa=1$.). This tendency is the same as in the case of the coincident-optimum-edge

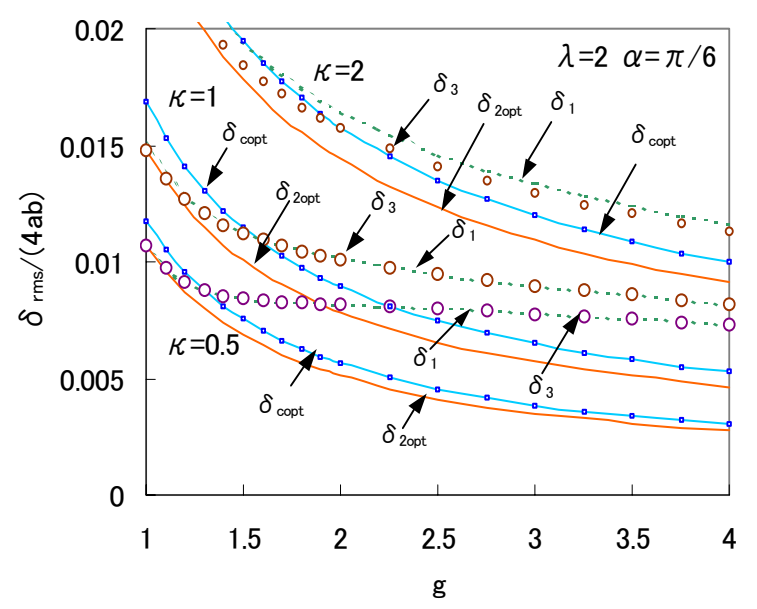

Fig. 8(b). Effects of $g$ upon the surface errors.

membrane of Ref. 19).

(3) When $(\lambda=1, g=1)$ or $(\lambda=1, g \neq 1, \alpha=\pi / 4)$, the surface errors are symmetric with respect to the centerline of $\kappa=1$ in the logarithmic axis of $\kappa$. When $\lambda=1, \alpha=0, g \neq 1$ and $\kappa$ is in the neighborhood of 1 , the surface errors of the optimized membranes become smaller as $\kappa$ decreases or as the tension applied in the direction parallel to the $\xi$ axis becomes higher.

(4) Each surface error of the optimized membranes for a slender configuration becomes smaller if the longer side of the rectangular membrane is loaded with higher tension and the shorter side is loaded with lower tension. The more slender the membrane is and the higher the ratio of tension (ratio of higher tension on the longer side to the lower tension on the shorter side) is, the smaller the surface error is.

(5) The surface error of each of the optimized membranes has a relative maximum or a relative minimum when the side is parallel to the direction of principal curvature at the point where the membrane is located. For a slender membrane, the minimum surface error is obtained when the longer side is parallel to the $\eta$ axis and the longer side is loaded with higher tension. For this slender membrane with a minimum surface error, each surface error has a minimum value at $\alpha=0 ; \delta_{\text {2opt }}$ and $\delta_{\text {copt }}$ have maximum values at $\alpha= \pm \pi / 2$; and $\delta_{1}$ and $\delta_{3}$ have maximum values at $\alpha \cong \pm \pi / 4$.

(6) It is found that the surface error of an optimum-2 membrane is the smallest among the optimized membranes, and the surface error of an optimum- 2 membrane $\delta_{2 \text { opt }}$ is about $0-15 \%$ less than that of the coincident-optimum-edge membrane $\delta_{\text {copt }}$ studied in Ref. 19).

(7) With the value of $\alpha=0, \delta_{\text {2opt }}$ is equal to $\delta_{3}$, and when $\lambda$ is large enough and $\kappa$ is small enough or $\lambda$ is small enough and $\kappa$ is large enough compared to $1, \delta_{2 \text { opt }}\left(=\delta_{3}\right)$ is approximately equal to $\delta_{1}$ and $\delta_{\text {copt }}$. With the value of $\alpha \neq 0$, when $\lambda$ is large enough and $\kappa$ is small enough or $\lambda$ is small enough and $\kappa$ is large enough compared to $1, \delta_{2 \text { opt }}$ $\left(\cong \delta_{\text {copt }}\right)$ is smaller than $\delta_{3}\left(\cong \delta_{1}\right)$. The ratio of $\delta_{\text {2opt }} / \delta_{3}$ is about 0.5 for $\alpha=\pi / 6, \pi / 4, \pi / 3$ and $\lambda=5,1 / 5$.

(8) Each surface error of the optimized membranes decreases as $g$ increases. When $\alpha$ is 0 , the decreasing rate of each optimized membrane is about the same. When $\alpha$ is not 0 , the decreasing 
Trans. JSASS Space Tech. Japan Vol. 4 (2006)

rates of $\delta_{1}$ and $\delta_{3}$ are less than those of $\delta_{2 \mathrm{opt}}$ and $\delta_{\text {copt }}$.

\section{Acknowledgments}

The author wishes to thank Prof. M.C. Natori of ISAS/JAXA (Institute of Space and Astronautical Science/ Japan Space Exploration Agency) and Emeritus Prof. Yuji Matsuzaki of Nagoya Univ. for their support of this research.

\section{References}

1) Headpeth, J. M.: New Concept for Precision Reflector Support Structure, IAF-85-208, 36th Congress of International Astronautical Federation, Stockholm, Oct. 1985.

2) Freeland, R. E.: Survey of Deployable Antenna Concepts, Large Space Antenna Systems Technology-1982, NASA-CP-2269, Part 1, May 1983, pp. 381-421.

3) Dyer, J. E.: Deployable Truss Structure Advanced Technology, First NASA/DOD CIS Technology Conference, NASA-CP-2447, Nov. 1986, pp. 111-124.

4) Mitsugi, J. and Yasaka, T.: A Modular Approach to Build a Large Space Antenna, IAF-91-315, 42nd Congress of the International Astronautical Federation, Montreal, 1991.

5) Miura, K., Inoue, T. and Tanizawa, K.: Structural Design of Tension Truss Antenna, IAF-91-316, 42nd Congress of the International Astronautical Federation, Montreal, 1991.

6) Baier, H., Datashvili, L., Gogava, Z., Medzmariashvili, E. and Montuori, V.: Building Blocks of Large Deployable Precision Membrane Reflectors, AIAA-2001-1478, AIAA/ASME/ASCE/AHS/ASC Structures, Structural Dynamics, and Materials Conference and Exhibit, April 2001, pp. 2205-2211.

7) Meguro, A., Harada, S. and Watanabe, M.: A High Precision Large Deployable Mesh Reflector for the Ku Band Mission, ISTS 2002-c-43, Proceedings of the 23rd International Symposium on Space Technology and Science, Vol. 1, 2002, pp. 622-627.

8) Bernasconi, M. C.: Large Spaceborn Antenna Reflector Using Inflatable Space Rigidized Structures, Proceedings of the Workshop on Mechanical Technology for Antennas, Noordwijk, ESA-SP-225, 1984, pp. 31-36.

9) Reibaldi, G. G and Bernasconi, M. C.: Quasat Program : the ESA Reflector,
IAF-85-400, 36th Congress of International Astronautical Federation, Stockholm, Oct. 1985.

10) Kato, S., Takeshita, Y., Sakai, Y., Muragishi, O., Shibayama, Y. and Natori, M.: Concept of Inflatable Elements Supported by Truss Structure for Reflector Application, Acta Astronautica, 19 (1989), pp. 539-553.

11) Natori, M., Kato, S., Muragishi, O. and Ohnishi, T.: Design and Development of a Modularized Inflatable Reflector, AIAA-90-0998, 31st AIAA/ASME/ ASCE/AHS/ASC Structures, Structural Dynamics and Materials Conference, Long Beach, CA, April 1990, pp. 2164-2172.

12) Freeland, R. E., Bilyeu, G D., Veal, G R. and Mikulas, M. M.: Inflatable Deployable Space Structure Technology Summary, IAF-98-I-5.01, 49th Congress of International Astronautical Federation, Oct. 1998.

13) Lichodziejewski, D. and Cassapaskis, C.: Inflatable Power Antenna Technology, AIAA-99-1074, 37th AIAA Aerospace Sciences Meeting and Exhibit, Reno, NV, Jan. 1999.

14) Fitcher, W. B.: Reduction of Root-Mean-Square Error in a Faceted Space Antennas, ALAAJ., 22 (1984), pp. 1679-1684.

15) Kato, S. and Natori, M.: Surface Accuracy Analysis of Mesh-Type Space Reflector -RMS Error of Circular and Equilateral Triangular Membrane Element-, Journal of the Japan Society for Aeronautical and Space Sciences, 41 (1993), pp. 283-292 (in Japanese).

16) Kato, S. and Matsuzaki, Y.: Surface Accuracy Analysis of Mesh-Type Space Reflector -RMS Error of a Rectangular Membrane Element-, Journal of the Japan Society for Aeronautical and Space Sciences, 42 (1994), pp. $98-107$ (in Japanese).

17) Furuya, H. and Miura, K.: Effects of Tension on Surface Accuracy of Membrane Space Antennas, Proceedings of JSASS/JSME 26th Structure Conference, Kobe, 1984, pp. 316-319 (in Japanese).

18) Furuya, H. and Miura, K.: Effects of Tension and Configuration in Surface Errors of Membrane Space Antennas, International Journal of Space Structures, 6(1991), pp. 219-226.

19) Kato, S.: Effects of Tension upon the Surface Accuracy of a Rectangular Membrane Element of a Space Reflector, Trans. JSASS Space Tech. Japan, 3 (2005), pp. 23-32.

20) Kato, S.: Surface Accuracy Analysis of an Inflatable Rectangular Membrane of a Space Reflector, Trans. JSASS Space Tech. Japan, 4 (2006), pp. 1-10.

21) Timoshenko, S. P. and Goodier, J. N.: Theory of Elasticity, Third Edition, International Student Edition, McGraw-Hill Kogakusha, Tokyo, 1970. 